\title{
Are high-frequency oscillations better biomarkers of the epileptogenic zone than spikes?
}

\author{
Nicolas Roehri ${ }^{1}$ and Fabrice Bartolomei ${ }^{1,2}$ \\ ${ }^{1}$ Aix Marseille Univ, Inserm, INS, Institut de Neurosciences des Systèmes \\ ${ }^{2}$ Clinical Neurophysiology and Epileptology Department, APHM, Timone Hospital, \\ Marseille, France
}

\begin{abstract}
Purpose of review

Precise localization of the epileptogenic zone is imperative for the success of resective surgery of drugresistant epileptic patients. To decrease the number of surgical failures, clinical research has been focusing on finding new biomarkers. For the past decades, high-frequency oscillations (HFOs, $80-500 \mathrm{~Hz}$ ) have ousted interictal spikes - the classical interictal marker - from the research spotlight. Many studies have claimed that HFOs were more linked to epileptogenicity than spikes. This present review aims at refining this statement in light of recent studies.

\section{Recent findings}

Analysis based on single-patient characteristics has not been able to determine which of HFOs or spikes were better marker of epileptogenic tissues. Physiological HFOs are one of the main obstacles to translate HFOs to clinical practice as separating them from pathological HFOs remains a challenge. Fast ripples (a subgroup of HFOs, 250-500Hz) which are mostly pathological are not found in all epileptogenic tissues.

Summary

Quantified measures of HFOs and spikes give complementary results, but many barriers still persist in applying them in clinical routine. The current way of testing HFO and spike detectors and their performance in delineating the epileptogenic zone is debatable and still lacks practicality. Solutions to handle physiological HFOs have been proposed but are still at a preliminary stage.

Keywords - classification, epilepsy, interictal spikes, pathological and physiological high-frequency oscillations, validation
\end{abstract}

\section{INTRODUCTION}

Drug-resistant focal epilepsies are devastating diseases for which surgery can be an effective treatment. In this context, presurgical investigations seek to determine the most epileptogenic regions of the patient's brain. Historically, these regions have been differentiated as the epileptogenic zone, brain regions generating seizures, and the irritative zone, the site of interictal epileptic discharges [1]. Complete removal of the epileptogenic zone is an essential factor

Correspondence to Fabrice Bartolomei, MD, PhD, Institut de Neurosciences des Systèmes, INS, UMR 1106, Faculté de Médecine La Timone, 27, bd Jean Moulin, 13005 Marseille, France. Tel: +33491384990;

e-mail: fabrice.bartolomei@ap-hm.fr in the disappearance of seizures after surgery [2]. Intracerebral recordings, in particular stereoelectroencephalography, are often required to define the epileptogenic regions. The epileptogenic zone definition is based on the recordings of seizures and particularly the site(s) of seizure onset (called the seizure onset zone, SOZ).

Interictal spikes are a marker of epileptogenicity but their predictive value of the epileptogenic zone is discussed. There are indeed possible dissociations between regions expressing the maximum number of spikes and those expressing the maximum epileptogenicity in a significant percentage of cases $(56 \%$ in [3]).

In the last 15 years, other markers of interictal 


\section{KEY POINTS}

- HFOs and spikes are complementary biomarkers of epileptogenic tissues.

- Distinguishing physiological from pathological HFOs remains challenging.

- HFO topography can be variable across nights and sleep stages.

- Multicentre study with automated HFO detectors has not been able to replicate previous findings.

activity have been proposed. Research has mainly concentrated on interictal high-frequency oscillations (HFOs). These small-amplitude oscillations between 80 and $500 \mathrm{~Hz}$ lasting more than four periods can be recorded using macroelectrodes or grids and are further divided into ripples $(80-250 \mathrm{~Hz})$ and fast ripples $(250-500 \mathrm{~Hz})-$ examples are illustrated in Fig. 1. Although ripples have been discovered under physiological conditions, fast ripples were only found in epileptogenic tissues [4].

Retrospective studies have shown that resection of areas with high rates of presurgical HFOs is associated with a better postsurgical seizure outcome [514]. This has led to place HFOs on a pedestal, despite having only a few studies, either chronic $[5,11,15-17]$ or intraoperative $[8,12]$ recordings, directly comparing HFOs to spikes.

Recent studies have, however, eroded the confidence in HFOs as being the modern biomarker of epilepsy. Correlation between surgical outcome and the amount of resected HFO generators (either ripples or fast ripples) has been difficult to replicate in a multicentre study using automated detection [18]. Physiological and pathological HFOs have similar characteristics characteristics which render their dichotomy delicate [19]. The HFO rate does not permit to delineate the SOZ more precisely than the rate of spikes [20]. Furthermore, fast ripples, whereas very specific to the $\mathrm{SOZ}$ are not sensitive enough $[20,21]$ and should be complementary targeted in intraoper- ative recordings after lesional resection [22]. This review aims at investigating these issues and discussing a paradigm shift for better and more robust use of HFOs and spikes in clinical practice.

\section{HIGH-FREQUENCY OS- CILLATIONS AND SPIKES ARE COMPLEMENTARY}

Early studies which have indicated that HFOs were better than spikes were mainly based on correlations between the amount of resected HFO areas and surgical outcome $[5,8,11]$. These results showed that the more HFOs are removed, the more likely it is for the patients to be seizure free. This was less clear with the spikes hence the alleged superiority of HFOs. Although these studies were necessary to build up interest in HFOs and to present statistical evidence concerning the link between HFOs and epileptogenicity, they are not sufficient to translate HFOs in clinical routine [24]. Indeed, these approaches summarize each patient into a single value and omit spatial concordance between the area generating HFOs and the epileptogenic zone. In other words, their benefit in planning the surgery is not tackled.To integrate the spatial extent of biomarkers, one should evaluate whether a given channel records from within the epileptogenic zone, that is whether this channel is situated inside the epileptogenic zone. One would use a binary classification method to label channels as inside or outside the epileptogenic zone which allows computing the sensitivity, specificity and precision of markers.

Although, in 2008, Jacobs et al. [15] already used this technique to promote the specificity of HFOs to the $\mathrm{SOZ}$, these results were calculated at the group level by taking the rates of all channels from the whole cohort of patients. This assumes that rates are comparable between patients and that a given threshold has the same meaning across patients. Moreover, clinicians face individual cases and thus need results that can be transposed to single patients. In a recent study [20], we therefore calculated the performance of HFO rate, its variants (ripples and fast ripples rates) 

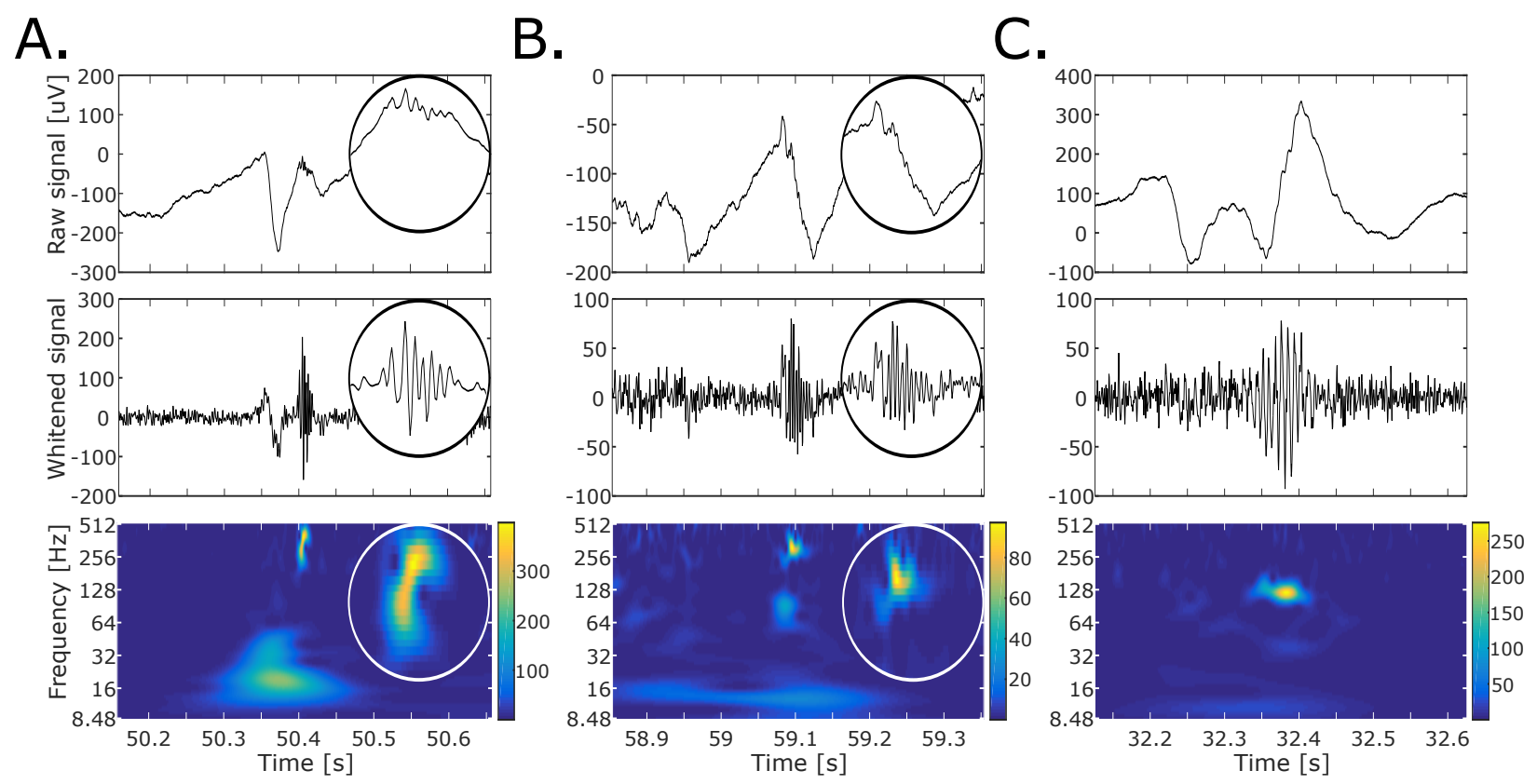

Figure 1: Examples of high-frequency oscillations (HFOs) in time and time-frequency domains. Top panels show HFOs in time domain, middle panels represent the whitened reconstruction of the respective HFOs and the bottom panel display the HFOs in the normalized/whitened time-frequency image. The whitening procedure used is the $\mathrm{zH} 0$ from Roehri et al. [23]. (a) Illustration of an interictal spikes preceding a fast ripples. (b) and (c) Example of a fast ripple and a ripple, respectively. The insets in a and b correspond to a zoomed version of the fast ripples in each domain. Source: Original.

and spike rate to predict a quantified estimation of the SOZ for each individual patient. Interestingly, neither HFOs nor its variants were better predictors of the SOZ than the spikes. We observed a variability from one patient to another, in some cases HFOs were performing better and in others the spikes were. (Fig. 2). The lack of specificity of spikes, as the irritative zone is usually broader than the $\mathrm{SOZ}$, and the presence of high rates of physiological HFOs weakened their respective performance.

In fact, HFOs and spikes are rather complementary because merging the two markers does outperform all other markers at both predicting the SOZ [20] and the outcome $[25,26]$. Unfortunately, the predictive performance was quite low for some patients (Fig. 2 ). An explanation could be that the irritative and HFO zones vary during hospitalization time and that the selected sleep section only partially reflected the epileptogenic zone. Indeed, both Fedele et al. [27] and Gliske et al. [28] showed that the HFO area could fluctuate across nights; the author did, however, not investigate the irritative zone which could change differently or possibly be more stable. Whether this is because of drug withdrawal, proximity to a given type of seizure onset or multiday rhythm [29] remains unknown. Moreover, a recent study from our group has shown that spikes increase during non-rapid eye movement sleep (NREM) sleep and that this increase was higher in medial temporal regions than in other regions, whether involved at seizure onset or not [30].

Future studies should focus on how to increase predicting values of spikes and HFOs rather than showing by mean of correlations that there is an effect between HFOs/spikes and epileptogenicity, which is 
now commonly admitted. Along with analyzing longterm traces, an approach could be to differentiate physiological from pathological HFOs as the advantage of pathological HFOs remains unclear.

\section{PHYSIOLOGICAL AND PATHOLOGICAL HIGH- FREQUENCY OSCILLA- TIONS}

Defining physiological HFOs is challenging because intracerebral electroencephalography (EEG) is not available in healthy study participants. Several options have been recently explored.

Frauscher et al. [31] have applied thorough criteria to define physiological channels in epileptic patients (i.e. channels in normal tissue(MRI), outside the $\mathrm{SOZ}$ and without interictal spikes or slow wave anomaly). Subsequently, HFOs automatically detected on these channels were labelled as physiological. By doing so, they produced a coarse atlas of physiological HFO rates along with regional normative values. The authors proposed that these normative values could be used in clinical practice as the value above which the rate is likely to be produced by pathological tissue $(\mathrm{p}<0.05)$. The authors showed that the highest rates of physiological ripples were recorded in the occipital cortex, medial and basal temporal region, transverse temporal gyrus and planum temporale, pre and postcentral gyri and medial parietal lobe. Interestingly, only a few fast ripples were detected and $5 \%$ of the channels had a fast ripples rate above $0.2 / \mathrm{min}$. The authors acknowledge that physiological regions defined as such is debatable but seems to be a good trade-off, and because some regions were sparsely sampled, more patients should be included to build a more robust atlas.

Although this effort in accounting for regional variability in HFO rates is pertinent, the capability of the normative values to delineate the pathological regions of a single patient has not yet been tested. Indeed, only the error of wrongly labelling a physiological channel as pathological is handled $(\mathrm{p}<0.05)$.
What should also be controlled is the sensitivity of this method, that is how many pathological channels are correctly labelled as pathological and wrongly labelled as physiological because they exhibited a rate below the normative value of the given region. Indeed, Guragain et al. [32] only found significant differences in $\mathrm{HFO}$ rates in $\mathrm{SOZ}$ compared to non-SOZ in parahippocampal, occipital and parietal regions. This could mean that in regions where these differences were not significant, the normative value would reject a great number of pathological tissues. On top of that, significant differences in rates do not ensure good separability between SOZ and non-SOZ.

Nevertheless, these two studies are different as one studied only recordings during NREM sleep stages $\mathrm{N} 2$ and N3 and physiological regions, whereas the other analyzed recordings between 1 and 3 a.m. and compared SOZ to non-SOZ (i.e. physiological areas plus regions in the irritative zone but outside the $\mathrm{SOZ}$ ). As it seems that the first sleep cycle is more appropriate to distinguish physiological from pathological HFOs because of distinct sleep-homeostatic properties [33], the absence of statistical evidence in some regions could be because of the fact that the authors did not study a consistent sleep stage. Nonetheless, accuracy of delineating epileptogenic tissues using this atlas has to be further quantified before using it in clinical routine.

Another approach to study physiological rhythms is to record epileptic patients during cognitive tasks inducing HFOs [34, 35] or implanted patients which do not suffer from epilepsy but another disease (controls) such as facial pain [19]. Characteristics of pathological, induced and control HFOs such as frequency, amplitude and duration are very alike which renders their classifications challenging [19]. It currently seems difficult to delineate the pathological from physiological tissues based on either the rate or features of HFOs. Instead of using only oscillatory features, analyzing more information could improve their separability.

Liu et al. [36] characterized HFOs based on the content of their detection instant (i.e. entropy, power ratio and main frequency). They proposed that pathological HFOs would occur preferentially in a similar context. Using unsupervised machine learning, they

Curr Opin Neurol 2019, 32:213-219

Author Version

DOI:10.1097/WCO.0000000000000663 


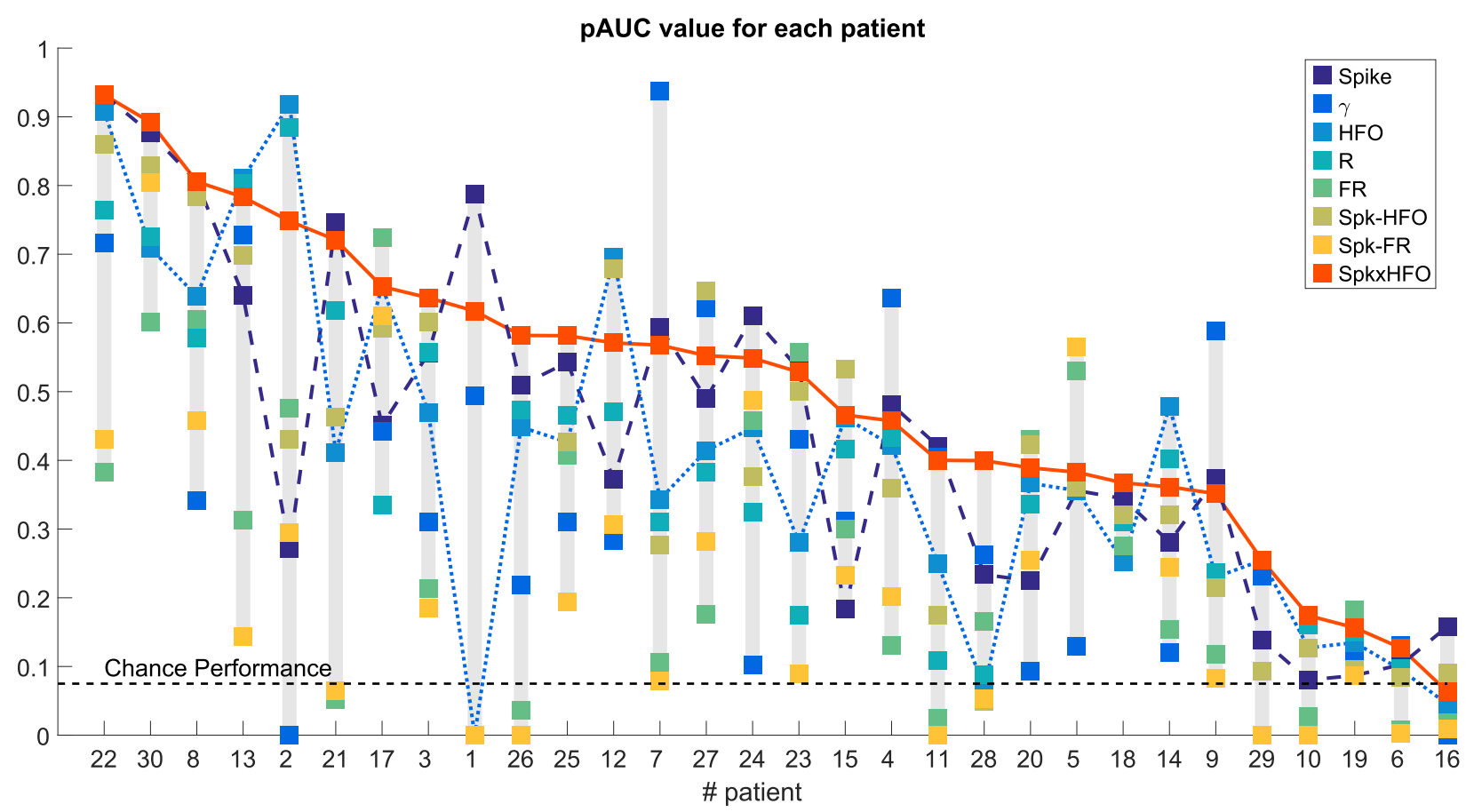

Figure 2: Partial AUC (pAUC) for each patient, ranked in decreasing order of the $\operatorname{Spk} \otimes$ HFO performance. pAUC is a performance metric which take into account sensitivity and specificity (here above $75 \%$ ) for different thresholds. Spk $\otimes$ HFO is obtained by multiplying the spike and the HFO rate. The solid, dotted, and dashed lines correspond to the performance of the Spk $\otimes$ HFOs, HFOs, and spikes, respectively, for each patient. The performance at chance level is 0.075 because of partial AUC analysis and normalization. AUC, area under the curve; HFO, high-frequency oscillation; g, rate of oscillations in the gamma band; Spk, spikes; Spk-HFO, rate of HFO co-occurring with a spike; Spk-FR, rate of fast ripples co-occurring with a spike. Adapted with permission from [20]

showed that indeed those 'stereotyped' HFOs tend to primarily arise from the SOZ and that HFOs in functional areas tend to appear in a more heterogeneous context. It is, however, not clear whether those 'stereotyped' HFOs correspond to the more commonly known HFOs riding spikes and whether the physiological ones represent the ones occurring without spikes. Moreover, the most stereotyped HFO clusters indicated the locations where the seizures mostly started but their sensitivity was quite low (24\%, ranging from 11 to $100 \%$ ), that is they were not found in the whole SOZ. These markers are thus interesting to point towards the epileptogenic

Curr Opin Neurol 2019, 32:213-219

DOI:10.1097/WCO.0000000000000663 region but not to fully delineate it.In fact, these sub-clusters mainly contained fast ripples (Supplementary Data [36]). Two recent independent studies $[20,21]$ showed that fast ripples, despite being specific to epileptogenic tissues, were only partially present in those tissues. Should we therefore continue chasing these very specific but not sensitive oscillations?

\section{CHASING FAST RIPPLES}

The amount of resected fast ripples is positively correlated with good outcome [5-11] and fast ripples re-
Author Version Page 5 
maining in postoperative recordings are mostly observed in patients with poor outcomes [12, 14, 22]. Complete removal of fast ripples thus seems to be imperative to achieve seizure freedom. However, many patients with bad outcome did not have residual fast ripples (15/24 in [12]). The authors hypothesized that it could be because of low spatial sampling. The two aforementioned stereoelectroencephalography studies [20, 21]rather suggest that fast ripples are only produced by a subset of the epileptogenic tissues (possibly the most epileptogenic ones). Therefore, resecting the remaining fast ripples may not guarantee seizure freedom, even though it could improve patient outcome.

In a multicentre study, Jacobs et al. [18] tried to replicate findings about fast ripples removal in a larger cohort of patients with automated detections. Although their results were concordant with the literature when analyzing data from the three centres together, they were unexpected at the centre level. Indeed, many patients from two centres had their ripple and fast ripples areas barely resected but were seizure free (minimum of 1-year follow-up). In a comprehensive and genuine discussion, the authors listed the possible confounders which yielded these results such as presence of physiological HFOs or the different resection strategies. Notably, the authors mentioned the use of an automated HFO detector which differs from their previous publications based on visual markings. They verified a posteriori that the detector performed correctly on a 1-min section. This, however, assumes that visual marking is the gold standard. Spring et al. [37] showed that the agreement of 6 reviewers was insufficient to reliably assess the presence of HFOs. They also estimated that 17 reviewers were required to have a strong agreement! Low interreviewer agreement also stands for spikes [38-40] even though the characteristic shape of the spike is more commonly accepted and there is no need of extreme band-pass filtering for reviewing them. Nevertheless, this type of agreement only considers whether a detection corresponds to a visually marked HFO but not whether it was correctly classified as ripples or fast ripples [41]. No detector has been tested on its classification capability. This possibly explains why so many detections labelled as fast ripples where not in the resected area; they could have been genuine ripples misclassified as fast ripples. Indeed, bandpass filters are not perfect filters. Some ripples with a frequency content near the limit of the bandpass are still visible in the fast ripples-band filtered signal[27]. To address this issue, a multicentre and open access effort has to emerge. Similarly to what was done with spindle detections [42], crowdsourced ripple and fast ripple identification could be used to create a gold standard data set for automated detection validation. Detectors performing well on such a data set would emulate human scoring. Nonetheless, true HFOs that appear unclear because of small amplitude or complicated background activity would still be missed. Alongside crowdsourced data sets, benchmark based on simulated signals $[41,43,44]$ could be used to characterize these detection types. Equivalent data sets have to be made for spike detections as interactions between HFOs and spikes are of interest.

The creation of such datasets is an essential step to build up robust and generalizable knowledge about HFOs and spikes. It is only after reaching this milestone that validated detectors can be applied to a large cohort of patients and their predictive performance evaluated. However, to be able to calculate such a performance, one has first to determine a gold standard that characterizes best best the epileptogenic zone.

\section{SEIZURE ONSET ZONE VS. RESECTED VOLUME}

It is commonly agreed that the SOZ is a biased estimation of the epileptogenic zone because it is an interpretation of a limited brain sampling and it is unknown whether the removal of the SOZ will ensure seizure freedom. Resected volume leading to a good postsurgical outcome is thus considered as the gold standard of the correct resection of the epileptogenic zone. As discussed above, only correlations to postsurgical outcome can be studied but what is currently needed is channel classification. This can be achieved with the resected volume.

By definition, an Engel I class patient had its

Curr Opin Neurol 2019, 32:213-219

Author Version

DOI:10.1097/WCO.0000000000000663 
epileptogenic zone resected. Nevertheless, the resected volume might not be exclusively composed of the epileptogenic zone. The amount of cortex which was mistakenly removed cannot be comprehended. Defining the sensitivity, specificity or precision of a given marker to the resected volume, as an estimation of the epileptogenic zone, is also biased and can only be calculated for patients with good outcome. For patients with bad outcome, clinicians may hypothesize that the epileptic focus was not entirely resected yet it could also be because the epileptogenic zone was not focal (which is the first criterion for resective surgery [45]) but rather an extended network. Contrariwise, if clinicians suspected an extended network, surgery may not have been carried out.

There is here a clear selection bias; the cohort of patient is not representative of the population intended to be analyzed. One should not compare biomarkers using only resected volume and generalize the results to focal refractory epilepsy as epileptogenic zones corresponding to extended networks or overlapping with eloquent areas were not studied. Some would argue that intracranial EEG suffers from selection bias as the whole brain is not sampled. This is however an intrinsic methodological bias, which researchers are well aware of, affecting both the SOZ and the resected volume. Furthermore, large explorations are more likely when extended networks are suspected. With such explorations, it is more likely to measure physiological HFOs and thus better appreciate the concordance of HFOs and the epileptogenic zone.

\section{CONCLUSION}

Recent findings have highlighted drawbacks of HFOs. These oscillations are not better than spikes to delineate the SOZ. Previous correlations with the outcome are difficult to replicate in cohort from multiple centres. Distinguishing physiological HFOs from pathological ones remain a challenge. The HFO rate is region-dependent hence the need of an atlas and the HFO area varies in time. For better reproducibility, gold standard data sets must be made to thoroughly

Curr Opin Neurol 2019, 32:213-219

DOI:10.1097/WCO.0000000000000663 validate $\mathrm{HFO}$ and spike detectors. Efforts to provide open access detectors and data sets have already been made but should be generalized (https: //mni-open-ieegatlas.research.mcgill.ca/; https://doi.org/10.6084/m9.figshare.4729645; http://dx.doi.org/10.6080/K06Q1VD5.). Correlation studies should evolve into studies that evaluate the predictive power of biomarkers. Finally, a global concertation needs to happen about how to properly assess the performance of HFOs and spikes in predicting the epileptogenic zone because both the SOZ and the resected volume are biased.

\section{Financial support and sponsor- ship}

This work has been carried out within the FHUEPINEXT with the support of the A*MIDEX project (ANR-11-IDEX-0001-02) funded by the 'Investissements d'Avenir' French Government program managed by the French National Research Agency (ANR). N.R. was supported by the French national clinical trial project SPREAD (PHRCN-160685 sponsored by University Hospitals of Strasbourg, IDRCB No. 2017-A00497-46).

\section{Conflicts of interest}

There are no conflicts of interest.

\section{References}

[1] Jean Talairach and Jean Bancaud. Lesion, "Irritative" Zone and Epileptogenic Focus. Confin. neurol, 27(1):91-94, 1966.

[2] Philippe Ryvlin, Helen Cross, and Sylvain Rheims. Epilepsy surgery in children and adults. The Lancet Neurology, 13(11):1114-1126, 2014. ISSN 1474-4422. doi: 10.1016/S1474-4422(14) 70156-5. URL www. thelancet.com/neurology.

[3] Fabrice Bartolomei, Agnes Trébuchon, Francesca Bonini, Isabelle Lambert, Mar-
Author Version Page 7 
tine Gavaret, Marmaduke Woodman, Bernard Giusiano, Fabrice Wendling, and Christian Bénar. What is the concordance between the seizure onset zone and the irritative zone? A SEEG quantified study. Clinical Neurophysiology, 127(2):1157-1162, 2016. ISSN 18728952. doi: 10.1016/j.clinph.2015.10.029. URL http://dx.doi.org/10.1016/j.clinph. 2015.10 .029 .

[4] A Bragin, J Engel, C L Wilson, I Fried, and G Buzsáki. High-frequency oscillations in human brain. Hippocampus, 9(2):137-142, 1999. ISSN 1050-9631. doi: 10.1002/(SICI)1098-1063(1999) 9:2〈137::AID-HIPO5 $>3.0 . C O ; 2-0$.

[5] Julia Jacobs, Maeike Zijlmans, Rina Zelmann, Claude Édouard Chatillon, Jeffrey Hall, André Olivier, François Dubeau, and Jean Gotman. High-frequency electroencephalographic oscillations correlate with outcome of epilepsy surgery. Annals of Neurology, 67(2):209-220, 2010. ISSN 03645134. doi: 10.1002/ana.21847.

[6] J. Y. Wu, R. Sankar, J. T. Lerner, J. H. Matsumoto, H. V. Vinters, and G. W. Mathern. Removing interictal fast ripples on electrocorticography linked with seizure freedom in children. Neurology, 75(19):1686-1694, 2010. ISSN 00283878. doi: 10.1212/WNL. 0b013e3181fc27d0.

[7] Tomoyuki Akiyama, Bláthnaid McCoy, Cristina Y. Go, Ayako Ochi, Irene M. Elliott, Mari Akiyama, Elizabeth J. Donner, Shelly K. Weiss, O. Carter Snead, James T. Rutka, James M. Drake, and Hiroshi Otsubo. Focal resection of fast ripples on extraoperative intracranial EEG improves seizure outcome in pediatric epilepsy. Epilepsia, 52(10):1802-1811, 2011. ISSN 00139580. doi: $\quad 10.1111 / \mathrm{j} .1528-1167.2011 .03199 . x$. URL http://doi.wiley.com/10.1111/j. 1528-1167.2011.03199.x.

[8] N. E C van Klink, M. A. van't Klooster, R. Zelmann, F. S S Leijten, C. H. Ferrier, K. P J Braun, P. C. van Rijen, M. J a M van Putten, G. J M Huiskamp, and M. Zijlmans. High frequency oscillations in intraoperative electrocorticography before and after epilepsy surgery. Clinical Neurophysiology, 125 (11):2212-2219, 2014. ISSN 13882457. doi: 10.1016/j.clinph.2014.03.004. URL http://dx. doi.org/10.1016/j.clinph.2014.03.004.

[9] Jounhong Ryan Cho, Dae Lim Koo, Eun Yeon Joo, Dae Won Seo, Seung-Chyul Hong, Premysl Jiruska, and Seung Bong Hong. Resection of individually identified high-rate high-frequency oscillations region is associated with favorable outcome in neocortical epilepsy. Epilepsia, 55 (11):1872-1883, 2014. ISSN 00139580. doi: 10. 1111/epi.12808. URL http://doi.wiley.com/ 10.1111/epi.12808.

[10] Karolin Kerber, Matthias Dümpelmann, Björn Schelter, Pierre Le Van, Rudolf Korinthenberg, Andreas Schulze-bonhage, and Julia Jacobs. Differentiation of specific ripple patterns helps to identify epileptogenic areas for surgical procedures. Clinical Neurophysiology, 125(7):13391345, 2014. ISSN 1388-2457. doi: 10.1016/j. clinph.2013.11.030. URL http://dx.doi.org/ 10.1016/j.clinph.2013.11.030.

[11] Claire Haegelen, Piero Perucca, Claude Edouard Châtillon, Luciana Andrade-Valença, Rina Zelmann, Julia Jacobs, D. Louis Collins, François Dubeau, André Olivier, and Jean Gotman. High-frequency oscillations, extent of surgical resection, and surgical outcome in drug-resistant focal epilepsy. Epilepsia, 54(5):848-857, 2013. ISSN 00139580. doi: 10.1111/epi.12075.

[12] Maryse A. van't Klooster, Nicole E C Van Klink, Frans S S Leijten, Rina Zelmann, Tineke A. Gebbink, Peter H. Gosselaar, Kees P J Braun, Geertjan J M Huiskamp, and Maeike Zijlmans. Residual fast ripples in the intraoperative corticogram predict epilepsy surgery outcome. $\mathrm{Neu}$ rology, 85(2):120-128, 2015. ISSN 1526632X. doi: 10.1212 /WNL.0000000000001727.

[13] Yvonne Höller, Raoul Kutil, Lukas Klaffenböck, Aljoscha Thomschewski, Peter M. Höller,

Curr Opin Neurol 2019, 32:213-219

Author Version

DOI:10.1097/WCO.0000000000000663 
Arne C. Bathke, Julia Jacobs, Alexandra C. Taylor, Raffaele Nardone, and Eugen Trinka. High-frequency oscillations in epilepsy and surgical outcome. A meta-analysis. Frontiers in Human Neuroscience, 9(October):1-14, 2015. ISSN 1662-5161. doi: 10.3389/fnhum.2015. 00574. URL http://journal.frontiersin. org/article/10.3389/fnhum. 2015.00574.

[14] Tommaso Fedele, Maryse Van, Sergey Burnos, Willemiek Zweiphenning, Maryse van't Klooster, Sergey Burnos, Willemiek Zweiphenning, Nicole van Klink, Frans Leijten, Maeike Zijlmans, and Johannes Sarnthein. Automatic detection of high frequency oscillations during epilepsy surgery predicts seizure outcome. Clinical Neurophysiology, 127(9):3066-3074, 2016. ISSN 13882457. doi: 10.1016/j.clinph.2016.06.009. URL http://linkinghub.elsevier.com/ retrieve/pii/S1388245716304394http:// dx.doi.org/10.1016/j.clinph.2016.06.009.

[15] Julia Jacobs, Pierre LeVan, Rahul Chander, Jeffery Hall, François Dubeau, and Jean Gotman. Interictal high-frequency oscillations (80$500 \mathrm{~Hz}$ ) are an indicator of seizure onset areas independent of spikes in the human epileptic brain. Epilepsia, 49(11):1893-1907, 2008. ISSN 00139580. doi: 10.1111/j.1528-1167.2008.01656. $\mathrm{x}$.

[16] Julia Jacobs, Pierre Levan, Claude Edouard Châtillon, Andr Olivier, Franois Dubeau, and Jean Gotman. High frequency oscillations in intracranial EEGs mark epileptogenicity rather than lesion type. Brain, 132(4):1022-1037, 2009. ISSN 00068950. doi: 10.1093/brain/awn351.

[17] M. Zijlmans, J. Jacobs, R. Zelmann, F. Dubeau, and J. Gotman. High-frequency oscillations mirror disease activity in patients with epilepsy. Neurology, 72(11):979-986, 2009. ISSN 00283878. doi: 10.1212/01.wnl.0000344402. 20334.81 .

[18] Julia Jacobs, Joyce Y. Wu, Piero Perucca, Rina Zelmann, Malenka Mader, Fran- cois Dubeau, Gary W. Mathern, Andreas Schulze-Bonhage, and Jean Gotman. Removing high-frequency oscillations. Neurology, page 10.1212/WNL.0000000000006158, 2018. ISSN 0028-3878. doi: 10. 1212/WNL.0000000000006158. URL http://www. neurology.org/lookup/doi/ 10.1212/WNL.0000000000006158.

[19] Jan Cimbalnik, Benjamin Brinkmann, Vaclav Kremen, Pavel Jurak, Brent Berry, Jamie Van Gompel, Matt Stead, and Greg Worrell. Physiological and pathological high frequency oscillations in focal epilepsy. Annals of Clinical and Translational Neurology, pages 1062-1076, 2018. ISSN 23289503. doi: 10.1002/acn3.618. URL http://doi.wiley.com/10.1002/acn3.618.

[20] Nicolas Roehri, Francesca Pizzo, Stanislas Lagarde, Isabelle Lambert, Anca Nica, Aileen McGonigal, Bernard Giusiano, Fabrice Bartolomei, and Christian-George Bénar. High-frequency oscillations are not better biomarkers of epileptogenic tissues than spikes. Annals of Neurology, 83:84-97, 2018.

[21] C. Cuello-Oderiz, N. von Ellenrieder, R. Sankhe, A. Olivier, J. Hall, F. Dubeau, and J. Gotman. Value of ictal and interictal epileptiform discharges and high frequency oscillations for delineating the epileptogenic zone in patients with focal cortical dysplasia. Clinical Neurophysiology, 129(6):1311-1319, 2018. ISSN 18728952. doi: $10.1016 / j$.clinph.2018.02.003. URL https: //doi.org/10.1016/j.clinph.2018.02.003.

[22] Maryse A. van 't Klooster, Nicole E.C. van Klink, Willemiek J.E.M. Zweiphenning, Frans S.S. Leijten, Rina Zelmann, Cyrille H. Ferrier, Peter C. van Rijen, Willem M. Otte, Kees P.J. Braun, Geertjan J.M. Huiskamp, and Maeike Zijlmans. Tailoring epilepsy surgery with fast ripples in the intraoperative electrocorticogram. Annals of Neurology, 81(5):664-676, 2017. ISSN 15318249. doi: 10.1002/ana.24928.

[23] Nicolas Roehri, Jean-Marc Lina, John C Mosher, Fabrice Bartolomei, and Christian-

Curr Opin Neurol 2019, 32:213-219

Author Version DOI:10.1097/WCO.0000000000000663 
George Bénar. Time-frequency strategies for increasing high frequency oscillation detectability in intracerebral EEG. IEEE Transactions on Biomedical Engineering, 63(12):2595-2606, 2016. ISSN 0018-9294. doi: 10.1109/TBME. 2016.2556425 .

[24] Jan Cimbalnik, Michal T. Kucewicz, and Greg Worrell. Interictal high-frequency oscillations in focal human epilepsy. Current Opinion in Neurology, 116(8):1477-1490, 2016. ISSN 1527-5418. doi: 10.1161/CIRCRESAHA.116.303790.The.

[25] Shuang Wang, Norman K. So, Bo Jin, Irene Z. Wang, Juan C. Bulacio, Rei Enatsu, Shenyi Dai, Zhong Chen, Jorge Gonzalez-Martinez, and Imad M. Najm. Interictal ripples nested in epileptiform discharge help to identify the epileptogenic zone in neocortical epilepsy. Clinical Neurophysiology, 128(6):945-951, 2017. ISSN 13882457. doi: 10.1016/j.clinph.2017.03. 033. URL http://linkinghub.elsevier.com/ retrieve/pii/S1388245717301219.

[26] Shennan Aibel Weiss, Brent Berry, Inna Chervoneva, Zachary Waldman, Jonathan Guba, Mark Bower, Michal Kucewicz, Benjamin Brinkmann, Vaclav Kremen, Fatemeh Khadjevand, Yogatheesan Varatharajah, Hari Guragain, Ashwini Sharan, Chengyuan Wu, Richard Staba, Jerome Engel, Michael Sperling, and Gregory Worrell. Visually validated semi-automatic high-frequency oscillation detection aides the delineation of epileptogenic regions during intra-operative electrocorticography. Clinical Neurophysiology, 129(10):2089-2098, 2018. ISSN 18728952. doi: 10.1016/j.clinph.2018. 06.030. URL https://doi.org/10.1016/j. clinph.2018.06.030.

[27] T Fedele, S Burnos, E Boran, N Krayenbuhl, P Hilfiker, T Grunwald, and J Sarnthein. Resection of high frequency oscillations predicts seizure outcome in the individual patient. Scientific Reports, 7(1):13836, 2017. ISSN 2045-2322. doi: 10.1038/s41598-017-13064-1.
[28] Stephen V. Gliske, Zachary T. Irwin, Cynthia Chestek, Garnett L. Hegeman, Benjamin Brinkmann, Oren Sagher, Hugh J. L. Garton, Greg A. Worrell, and William C. Stacey. Variability in the location of high frequency oscillations during prolonged intracranial EEG recordings. Nature Communications, 9(1): 2155, 2018. ISSN 2041-1723. doi: 10.1038/ s41467-018-04549-2. URL http://www. nature. com/articles/s41467-018-04549-2.

[29] Maxime O. Baud, Jonathan K. Kleen, Emily A. Mirro, Jason C. Andrechak, David KingStephens, Edward F. Chang, and Vikram R. Rao. Multi-day rhythms modulate seizure risk in epilepsy. Nature Communications, 9 (1):1-10, 2018. ISSN 20411723. doi: 10. 1038/s41467-017-02577-y. URL http://dx. doi.org/10.1038/s41467-017-02577-y.

[30] Isabelle Lambert, Nicolas Roehri, Bernard Giusiano, Romain Carron, Fabrice Wendling, Christian Benar, and Fabrice Bartolomei. Brain regions and epileptogenicity influence epileptic interictal spike production and propagation during NREM sleep in comparison with wakefulness. Epilepsia, 59(1):235-243, 2018. ISSN 15281167. doi: $10.1111 /$ epi.13958.

[31] Birgit Frauscher, Nicolas Von Ellenrieder, Rina Zelmann, Irena Doležalová, Lorella Minotti, André Olivier, Jeffery Hall, Dominique Hoffmann, Dang Khoa Nguyen, Philippe Kahane, François Dubeau, and Jean Gotman. Atlas of the normal intracranial electroencephalogram: Neurophysiological awake activity in different cortical areas. Brain, 141(4):1130-1144, 2018. ISSN 14602156. doi: 10.1093/brain/awy035.

[32] Hari Guragain, Jan Cimbalnik, Matt Stead, David M. Groppe, Brent M. Berry, Vaclav Kremen, Daniel Kenney-Jung, Jeffrey Britton, Gregory A. Worrell, and Benjamin H. Brinkmann. Spatial variation in high-frequency oscillation rates and amplitudes in intracranial EEG. $\mathrm{Neu}$ rology, page 10.1212/WNL.0000000000004998, 2018. ISSN 0028-3878. doi: 10.1212/ 
WNL.0000000000004998. URL http://www. ncbi.nlm.nih.gov/pubmed/29367441; http: //www.neurology.org/lookup/doi/10.1212/ WNL. 0000000000004998.

[33] Nicolás von Ellenrieder, François Dubeau, Jean Gotman, and Birgit Frauscher. Physiological and pathological high-frequency oscillations have distinct sleep-homeostatic properties. NeuroImage: Clinical, 14:566-573, 2017. ISSN 22131582. doi: 10.1016/j.nicl.2017.02. 018. URL http://linkinghub.elsevier.com/ retrieve/pii/S2213158217300529.

[34] A. Matsumoto, B. H. Brinkmann, S. Matthew Stead, J. Matsumoto, M. T. Kucewicz, W. R. Marsh, F. Meyer, and G. Worrell. Pathological and physiological high-frequency oscillations in focal human epilepsy. Journal of Neurophysiology, 110(8):1958-1964, 2013. ISSN 0022-3077. doi: 10.1152/jn.00341. 2013. URL http://jn.physiology.org/cgi/ doi/10.1152/jn.00341.2013.

[35] M. T. Kucewicz, J. Cimbalnik, J. Y. Matsumoto, B. H. Brinkmann, M. R. Bower, V. Vasoli, V. Sulc, F. Meyer, W. R. Marsh, S. M. Stead, and G. a. Worrell. High frequency oscillations are associated with cognitive processing in human recognition memory. Brain, 137(8):2231-2244, 2014. ISSN 00068950. doi: 10.1093/brain/awu149. URL http://www . brain. oxfordjournals .org/ cgi/doi/10.1093/brain/awu149.

[36] S Liu, C Gurses, Z Sha, MM Quach, A Sencer, and N Bebek Brain. Stereotyped high-frequency oscillations discriminate seizure onset zones and critical functional cortex in focal epilepsy. Brain : a journal of neurology, (February):1-18, 2018. ISSN 0006-8950. doi: 10.1093/brain/awx374. URL https://academic.oup.com/brain/ advance-article/doi/10.1093/brain/ awx374/4831041.

[37] Aaron M. Spring, Daniel J. Pittman, Yahya Aghakhani, Jeffrey Jirsch, Neelan Pillay, Luis E. Bello-Espinosa, Colin Josephson, and Paolo
Federico. Generalizability of High Frequency Oscillation Evaluations in the Ripple Band. Frontiers in Neurology, 9(June):1-11, 2018. ISSN 1664-2295. doi: 10.3389/fneur.2018. 00510. URL https://www.frontiersin.org/ article/10.3389/fneur.2018.00510/full.

[38] Matthias Dümpelmann and C E Elger. Visual and automatic investigation of epileptiform spikes in intracranial EEG recordings. Epilepsia, 40(3):275-85, 1999. ISSN 0013-9580. URL http: //www.ncbi.nlm.nih.gov/pubmed/10080505.

[39] Daniel T Barkmeier, Aashit K Shah, Danny Flanagan, Marie D Atkinson, Rajeev Agarwal, Darren R Fuerst, Kourosh Jafari-khouzani, and Jeffrey A Loeb. High inter-reviewer variability of spike detection on intracranial EEG addressed by an automated multi-channel algorithm. Clinical Neurophysiology, 123(6):10881095, 2012. ISSN 1388-2457. doi: 10.1016/j. clinph.2011.09.023. URL http://dx.doi.org/ 10.1016/j.clinph.2011.09.023.

[40] Mark L. Scheuer, Anto Bagic, and Scott B. Wilson. Spike detection: Inter-reader agreement and a statistical Turing test on a large data set. Clinical Neurophysiology, 128(1):243-250, 2016. ISSN 13882457. doi: 10.1016/j.clinph.2016.11. 005. URL http://linkinghub.elsevier.com/ retrieve/pii/S1388245716306502.

[41] Nicolas Roehri, Francesca Pizzo, Fabrice Bartolomei, Fabrice Wendling, and ChristianGeorge Bénar. What are the assets and weaknesses of HFO detectors? A benchmark framework based on realistic simulations. PLoS ONE, 12(4):e0174702, apr 2017. ISSN 1932-6203. doi: 10.1371/journal.pone.0174702. URL http://dx.plos.org/10.1371/journal. pone. 0174702 .

[42] Simon C. Warby, Sabrina L. Wendt, Peter Welinder, Emil G.S. Munk, Oscar Carrillo, Helge B.D. Sorensen, Poul Jennum, Paul E. Peppard, Pietro Perona, and Emmanuel Mignot. Sleep-spindle detection: Crowdsourcing and evaluating performance of experts, non-experts

Curr Opin Neurol 2019, 32:213-219

Author Version DOI:10.1097/WCO.0000000000000663 
and automated methods. Nature Methods, 11(4): 385-392, 2014. ISSN 15487105. doi: 10.1038/ nmeth.2855.

[43] Gwénaël Birot, Amar Kachenoura, Laurent Albera, Christian Bénar, and Fabrice Wendling. Automatic detection of fast ripples. Journal of Neuroscience Methods, 213(2):236-249, 2013. ISSN 01650270. doi: 10.1016/j.jneumeth.2012. 12.013. URL http://dx.doi.org/10.1016/j. jneumeth.2012.12.013.

[44] Nisrine Jrad, Amar Kachenoura, Isabelle Merlet, Fabrice Bartolomei, Anca Nica, Arnaud Biraben, and Fabrice Wendling. Automatic detection and classification of High Frequency Oscillations in depth-EEG signals. IEEE Transactions on Biomedical Engineering, $\operatorname{PP}(99): 1-1$, 2016. ISSN 0018-9294. doi: 10.1109/TBME. 2016.2633391. URL http://ieeexplore.ieee. org/document/7762043/.

[45] Philippe Kahane, Elisabeth Landré, Lorella Minotti, Stafano Francione, and Philippe Ryvlin. The Bancaud and Talairach view on the epileptogenic zone: A working hypothesis. Epileptic Disorders, 8(SUPPL. 2):16-26, 2006. ISSN 12949361.

Curr Opin Neurol 2019, 32:213-219

Author Version DOI:10.1097/WCO.0000000000000663 